\title{
Compact symmetric Poisson equation discretization for non-hydrostatic sigma coordinates ocean model
}

\author{
Roullet Guillaume ${ }^{1,{ }^{*}}$, Molemaker Maarten Jeroen ${ }^{1,2}$, Ducousso Nicolas ${ }^{1}$, Dubos Thomas ${ }^{3}$
}

${ }^{1}$ Univ. Brest, CNRS, IRD, Ifremer, Laboratoire d'Océanographie Physique et Spatiale, IUEM, Brest, France.

${ }^{2}$ UCLA, Los Angeles, USA

3 IPSL/Lab. de Météorologie Dynamique, École Polytechnique, Palaiseau, France

*Corresponding author : Guillaume Roullet, email addresses : roullet@univ-brest.fr ; guillaume.roullet@univ-brest.fr

\begin{abstract}
:
In anticipation of relaxing the hydrostatic assumption in a sigma coordinates primitive equations ocean model, we show how a projection method can be designed with the use of a compact symmetric 15point stencil for the Poisson equation. This is achieved by recognizing that, owing the non-orthogonality of the grid, the velocity has a contravariant and covariant set of components. The two sets play a different role in the primitive equations: the contravariant components enter the definition of the model fluxes, whereas the covariant components experience the forces and in particular the pressure gradient. By treating these two sets separately, the discretized gradient and divergence operators are simple finite differences. The two sets of components are related via a linear transformation, the metric tensor, which is entirely determined by the kinetic energy. We show how the spatial discretization of the kinetic energy fully controls the Poisson equation discretization, including its boundary conditions. The discretization of the Poisson equation is shown to converge at second order and to behave as well or better than alternative methods. This approach is a prerequisite to implement later an efficient Poisson solver, such as a multigrid algorithm.
\end{abstract}

\section{Highlights}

A new formulation of the non-hydrostatic primitive equations in sigma coordinates is proposed. Momentum and flux are two different objects related via a linear transformation. - The Poisson equation for pressure is discretized with a compact symmetric stencil. The approach is a prerequisite to implement a fast Poisson solver for a forthcoming non-hydrostatic version of ROMS.

Keywords : Non-hydrostatic, Sigma coordinates, Poisson equation 
With the growing interest on processes at submesoscale (Nikurashin et al., 3 2013; Molemaker et al., 2015; Callies et al., 2015; Gula et al., 2015) the ocean 4 modelling community needs non-hydrostatic $(\mathrm{NH})$ ocean circulation models. 5 This will allow to investigate in realistic contexts the full range of internal 6 waves, symmetric instability, convection processes, etc. and to bridge the gap 7 with LES (Sullivan et al., 1994). A few circulation models do already handle 8 the non-hydrostatic physics properly: MITgcm (Marshall et al., 1997), POM 9 (Kanarska and Maderich, 2003), ROMS (Kanarska et al., 2007), Symphonie 10 (Auclair et al., 2011), GETM (Klingbeil and Burchard, 2013). However, NH 11 simulations on large grids, in realistic regional configurations are still awaited. 12 It is timely to resolve this. The reason for the NH circulation models rarity 13 is that relaxing the hydrostatic assumption, while keeping the Boussinesq 14 assumption, is not straightforward. At least two levels of difficulties must 15 be faced: i) the requirement to solve a 3D Poisson equation for the pressure 16 at each time step, made more complex in the case of sigma coordinates by 17 ii) the non-orthogonality of the grid. This second difficulty is absent in $z$ ${ }_{18}$ coordinates models. Incidentally, for models with a time-splitting on the 19 free surface, a third difficulty is to properly handle the coupling between the 

${ }_{43}$ an iterative solver to solve the Poisson equation. Many different numerical

The first problem amounts to solving a linear system of equations with as many equations as number of grid points. For the intended purpose of high-resolution turbulent simulations we have in mind grids as large as $2000 \times 2000 \times 1000$ grid points, corresponding to $N \sim 10^{9}$ coupled equations. It is a classical well known problem of High Performance Computing (HPC). A possible way to circumvent the problem consists in relaxing the incompressibility constraint, which forces to cope with sound waves. The pressure can then be computed by integrating in time the compressible physics using a time-splitting technique. In the atmospheric community, this is the approach implemented in WRF (Skamarock and Klemp, 2008). In the ocean the ratio between advective speed and the phase speed of sound waves is much more unfavorable, leading to a required time split that is two orders of magnitude larger. An approach that uses artificial impressibility to slow down the phase speed of sound waves and hence reduce the required ratio in split time steps is currently under development as part of the French regional ocean modeling project CROCO, but not available in a published manuscript (Francis Auclair, private communication). Klingbeil and Burchard (2013) follow a similar approach where a time-splitting on the baroclinic time step is introduced to achieye the incompressibility constraint. These methods trade the price of solving the Poisson problem with accepting a non-strict divergence-freeness of the flow.

Otherwise, the numerical implementation necessarily requires the use of methods exist but only a few of them succeed at maintaining a uniform

non-hydrostatic pressure and surface waves (Auclair et al., 2011). 
${ }_{68}$ 2003) resulting in wide stencils for the gradient operator. The divergence of 69

convergence rate for large grids. This is the case of the multigrid technique whose computational cost scales as $N \log N$, which is considered to be the optimal scaling. It has been shown recently to perform very well with large grids on massively parallel cluster, up to $10^{10}$ degrees of freedom and 65536 cores, in the context of atmospheric modelling (Müller and Scheichl, 2014). To get the best performances, the stencil for the Poisson equation needs to be symmetric and compact. These two properties reduce the number of floating point operations, the amount of data storage and the data movement between the cpu and the memory. In modern computer architecture, data movement is the bottleneck for high performances (Williams et al., 2009). Any numerical method that reduce data transfer is worth it. In the present study, the compactness means a 15-point stencíl, compared to the usual 25point one (Auclair et al., 2011). The symmetry allows to almost halve the number of coefficients for the matrix since only 8 coefficients are needed for a 15-point stencil (one main and 7 lower diagonals). Overall the present matrix offers a reduction by a factor 3 on the number of matrix coefficients compared to existing methods. In anticipation of implementing such solution into a sigma-cøordinates model we present here a way to achieve compactness and symmetry for the Poisson equation.

The second problem is due to the non-orthogonality of the grid. One consequence is that the horizontal pressure gradient can not be computed as a simple finite difference between two adjacent horizontal grid cells. Chain rule and vertical interpolation should be used (Shchepetkin and McWilliams, vertical flux behaves similarly because the vertical flux involves horizontal 

${ }_{93}$ the covariant components should be discretized at the edges of the dual grid

${ }_{94}$ (the lines joining the cell centers). Such discretization is in line with the

velocities. Since the Poisson equation arises as the successive action of the gradient and the divergence, the resulting stencil for the Poisson equation is tall in the vertical, involving overall 25 points (Auclair et al., 2011). The Poisson equation couples points over 5 levels in the vertical and 3 in both horizontal directions. The stencil can be made symmetric by defining the gradient as minus the adjoint of the divergence. This is the so-called compatible discretization (Taylor and Fournier, 2010). It is also possible to discretize the Poisson matrix directly and independently of how the gradient and the divergence are discretized (Kanarska et al., 2007). In that case, the stencil is compact with 15 points. The major drawback of such a method is that it fails at maintaining the compatibility between operators which impacts the energy conservation.

In this paper we show how the two problems can be solved jointly. The first step is to recognize that because of the non-orthogonality of the coordinates system, the velocity has two sets of components: a contravariant one and a covariant one. The two sets are related via a linear transformation, the metric tensor, which is invertible. The metric tensor is completely determined by the kinetic energy. The two sets of components play a different role and must be treated distinguishly. The contravariant components appear in the definition of fluxes (volume, tracer, momentum), whereas the covariant components appear in the force budget. Using the framework of the discrete differential geometry (Desbrun et al., 2008) we show that the contravariant components must be discretized at the cell faces of the primal grid whereas
(the lhies joining the cell centers). Such discretization is in line with the 
C-grid staggering, and the finite volume discretization for tracers. It also completely adheres to Thuburn and Cotter (2012) way to discretize shallow water equations on non-orthogonal grids. The divergence and the gradient operators have then trivial discretization: they are simple finite differences. In the spirit of Molemaker et al. (2005) and Dubos et al. (2015) we then deduce the discrete Poisson equation directly from the discretized kinetíc energy and the discretized divergence operator. The price of this approach is to prognose either the covariant components or the contravariant ones. For sake of completeness we present the momentum equations written for the covariant components in both vector-invariant form and in flux-form. The vector-invariant form is similar to a standard Cartesian formulation whereas the flux-form involves additional pseudo-force terms. Up to now, sigmacoordinates models prognose Cartesian components of the velocity, namely horizontal and vertical, which are a blend of contravariant and covariant components. This causes unnecessary wide stencil for the divergence and the gradient and finally a too wide stencil for the Poisson equation. By expliciting and clarifying the subtleties induced by non-orthogonal coordinates, this paper offers a roadmap for an efficient implementation of a projection method.

The paper is organized as follows. We define in section 2 the contraand covariant sets of components for the velocity and present the model continuous equations. In section 3 , we present the spatial discretization of the variables, the divergence and the discretized kinetic energy and derive all the other discretizations from them. In section 4 we discuss the properties of the discretization on a test-case. A conclusion is given in section 5 . 


\section{Formulation}

As stated in the introduction, the objective of this study is to provide a discrete Poisson operator that can be solved efficiently by iterative methods on very large grids. In this section, we will introduce a dual representation of the velocities on the grid, which will prove to be advantageous in arriving at a compact, symmetric, discrete operator. Subsequently, we will present the governing equations of motion for these prognostic variables.'

\subsection{Flux and momentum}

In orthogonal curvilinear coordinates $(\xi, \eta, z)$, the velocity of a fluid parcel is naturally expressed as $\mathbf{u}=h_{\xi} \dot{\xi} \mathbf{i}+h_{\eta} \dot{\eta} \mathbf{j}+\dot{z} \mathbf{k}$, where the dot denotes the Lagrangian time derivative, $\mathbf{i}, \mathbf{j}, \mathbf{k}$ are the unit vectors in horizontal and vertical directions, and $\left(h_{\xi}, h_{\eta}\right)$ are the Lamé coefficients in the horizontal directions. We define $\left(u_{c}, v_{c}, w_{c}\right)=\left(h_{\xi} \dot{\xi}, h_{\eta} \dot{\eta}, \dot{z}\right)$ and denote them, with a slight abuse of usage, the Cartesian velocity components. The terrain following sigma coordinates are $(\xi, \eta, \sigma)$, for which the vertical position is $z(\xi, \eta, \sigma, t)$. The time dependency in $z$ is driven by the sea surface height variations and yields a breathing grid. Using the chain rule, this causes

$$
\mathbf{u}=u_{c} \mathbf{i}+v_{c} \mathbf{j}+\left(u_{c} s_{\xi}+v_{c} s_{\eta}+h_{\sigma} \dot{\sigma}+\partial_{t} z\right) \mathbf{k},
$$

$$
s_{\xi}=\frac{1}{h_{\xi}} \frac{\partial z}{\partial \xi}, \quad s_{\eta}=\frac{1}{h_{\eta}} \frac{\partial z}{\partial \eta}
$$

are the slopes of $\sigma$-surfaces, $h_{\sigma}$ is the Lamé coefficient in the vertical direction ${ }_{139}$ and $\partial_{t} z=\partial z / \partial t$ accounts for the breathing of grid. We denote $(U, V, W)=$ 
$\left(h_{\xi} \dot{\xi}, h_{\eta} \dot{\eta}, h_{\sigma} \dot{\sigma}\right)$ the sigma coordinate velocity components. They are related to the Cartesian velocity components by

$$
\begin{aligned}
& u_{c}=U \\
& v_{c}=V \\
& w_{c}=U s_{\xi}+V s_{\eta}+W+\partial_{t} z .
\end{aligned}
$$

$$
W^{a}=W+\partial_{t} z=w_{c}-U s_{\xi}-V s_{\eta}
$$

the vertical velocity relative to a fixed grid, from $W$, the vertical velocity through the breathing grid. In the sequel, we refer to $\left(U, V, W^{a}\right)$ as the contravariant components of velocity. Notice that it is a slight abuse of language, since strictly speaking the contravariant components of velocity are just the material derivatives of the coordinates, i.e. $(\dot{\xi}, \dot{\eta}, \dot{\sigma})$, while $\left(U, V, W^{a}\right)$ incorporate the Lamé coefficients $\left(h_{\xi}, h_{\eta}, h_{\sigma}\right)$. With this definition, $\left(U, V, W^{a}\right)$ are close to the Cartesian/components typically manipulated by existing hydrostatic models, which simplifies the implementation of our method in such models. A similar cayeat applies to the covariant velocity components defined below.

The velocity can be written in vectorial form as

$$
\mathbf{u}=U\left(\mathbf{i}+s_{\xi} \mathbf{k}\right)+V\left(\mathbf{j}+s_{\eta} \mathbf{k}\right)+W^{a} \mathbf{k}
$$

for which it is clear that $\left(\mathbf{i}+s_{\xi} \mathbf{k}, \mathbf{j}+s_{\eta} \mathbf{k}, \mathbf{k}\right)$ are the basis vectors aligned with ${ }_{155}$ the coordinates $(\xi, \eta, \sigma)$. Interestingly there are two possible interpretations for the contravariant components: i) according to (3) and (4), $U$ and $V$ are 
horizontal components and $W^{a}$ is the vertical component normal to sigma isosurfaces ; ii) according to (5), $U$ and $V$ are along coordinates lines and $W^{a}$ is the vertical component. This dual interpretation is a source of confusion. The first interpretation is motivated by discrete differential geometry (Desbrun et al., 2008), the second interpretation is more in line with the physicist way of seeing the contravariant components. In this paper we will adopt the first interpretation and motivate it more thoroughly in section 3 .

Now that we have the contravariant components of the velocity we can define the covariant components ${ }^{1}$. For that we use the kinetic energy $K$ as a natural inner product, allowing to define the metric tensor. In terms of $\left(U, V, W^{a}\right) K$ reads

$$
K=\frac{1}{2}\left[U^{2}+V^{2}+\left(U s_{\xi}+V s_{\eta}+W^{a}\right)^{2}\right]
$$

where we have used $W^{a}$, not $\widehat{W}$, because the grid motion should not be accounted for in the kinetic energy. The covariant set of components $(u, v, w)$ is defined such that

$$
K=\frac{1}{2}\left(u U+v V+w W^{a}\right) .
$$

\footnotetext{
${ }^{1}$ As for the contravariant velocity components, our definition of covariant components is slightly abusive. The standard covariant components are obtained by multiplying ours by the corresponding Lamé coefficients. Again, our definition results in $(\mathrm{u}, \mathrm{v}, \mathrm{w})$ being closer to the Cartesian components, simplifying the implementation in existing hydrostatic models while ensuring key relationships $(7,8)$.
} 
171 We can find these components by differentiating $K$ with respect to $U, V, W^{a}$

$$
\begin{aligned}
& u=\partial K / \partial U=U\left(1+s_{\xi}^{2}\right)+V s_{\xi} s_{\eta}+W^{a} s_{\xi} \\
& v=\partial K / \partial V=U s_{\xi} s_{\eta}+V\left(1+s_{\eta}^{2}\right)+W^{a} s_{\eta} \\
& w=\partial K / \partial W^{a}=U s_{\xi}+V s_{\eta}+W^{a} .
\end{aligned}
$$

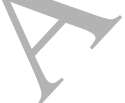

$$
\begin{aligned}
& u=u_{c}+w_{c} s_{\xi} \\
& v=v_{c}+w_{c} s_{\eta} \\
& w=w_{c} .
\end{aligned}
$$

We may use these components to rewrite the velocity in vectorial form

$$
\mathbf{u}=u \mathbf{i}+\nu \mathbf{j}+w\left(\mathbf{k}-s_{\xi} \mathbf{i}-s_{\eta} \mathbf{j}\right)
$$

Again we have a double interpretation for the covariant components: i) according to $(9)(u, v, w)$ are aligned with the coordinates lines and in particular $w$ is the vertical component; ii) according to $(10),(u, v, w)$ are orthogonal to coordinates lines. Tó be consistent with our previous choice, we need to chose the first interpretation.

The system (8) defines the metric tensor relating contravariant to covariant components. It is a $3 \times 3$ full matrix. Interestingly the inverse transformation has less entries

$$
\begin{aligned}
U & =u-w s_{\xi} \\
V & =v-w s_{\eta} \\
W^{a} & =-u s_{\xi}-v s_{\eta}+w\left(1+s_{\xi}^{2}+s_{\eta}^{2}\right) .
\end{aligned}
$$


183

We define

$$
\mathbf{T}=\left(\begin{array}{ccc}
1 & 0 & -s_{\xi} \\
0 & 1 & -s_{\eta} \\
-s_{\xi} & -s_{\eta} & 1+s_{\xi}^{2}+s_{\eta}^{2}
\end{array}\right)
$$

the matrix transforming $(u, v, w)$ into $\left(U, V, W^{a}\right)$. It can be used to express the kinetic energy

$$
K=\frac{1}{2}(u, v, w) \mathbf{T}(u, v, w)^{T},
$$

seen as an inner product between covariant components. The $\mathbf{T}$ matrix is the inverse of the metric tensor, it plays a central role in the discretization we propose.

At this stage we have set the framework. We have three different sets of velocity components available: Cartesian, contravariant and covariant. The Cartesian one is appealing but it does not correspond to either the coordinates lines or to the lines orthogonal to the coordinates hyperplanes. By contrast the contravariant and the covariant sets are natural. None of them is superior and they both have their interest. The covariant components are the natural prognostic variables of the momentum equations, while the contravariant components appear in all the fluxes and in particular into the incompressibility equation. For these reasons, $(u, v, w)$ will be called momentum and $\left(U, V, W^{a}\right)$ flux. In this approach flux and momentum are clearly two different objects. They handle their "horizontal" and "vertical" components very differently.

Finally because the two objects are related by the kinetic energy, their relation depends on whether the hydrostatic assumption is made or not. 
203

Within the hydrostatic assumption, $K$ simplifies into

$$
K_{\text {hydro }}=\frac{1}{2}\left[U^{2}+V^{2}\right] \text {. }
$$

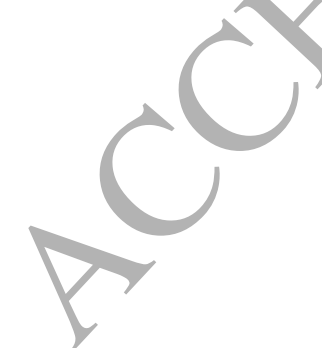

219 220 issue.

This implies $w=0$, which expresses the fact that the vertical momentum equation is a diagnostic equation. It also implies $(u, v)=(U, V)=\left(u_{c}, v_{c}\right)$, which means that the distinction between contravariant and covariant vanishes. Therefore everything can be cast in terms of Cartesian components and these subtleties can be ignored. The case of $W^{a}$ is special because it keeps its definition (4), albeit with $w=0$. $W$, the flux through the moving grid, is called the omega velocity. The distinction between Cartesian, contravariant and covariant components is thus essentially a non-hydrostatic

\subsection{Equations in momentum variables}

We now derive the momentum equations for the newly defined momentum components $(u, v, w)$, used as prognostic variables. As starting point, we will repeat equations $(3.8-3.10)$ together with equations $(3.13)$ and $(3.16)$ from Kanarska et al. (2007) that define the momentum equations in terms of substantial derivatives of Cartesian velocity components, i.e. in flux form,

$$
\begin{aligned}
& \frac{\mathcal{D} u_{c}}{\mathcal{D} t}=-\left.\mathcal{A}_{\xi} \frac{\partial\left(p^{h}+q\right)}{\partial \xi}\right|_{z}+\mathcal{V}\left(C_{u_{c}}+G_{u_{c}}+F_{u_{c}}\right) \\
& \frac{\mathcal{D} v_{c}}{\mathcal{D} t}=-\left.\mathcal{A}_{\eta} \frac{\partial\left(p^{h}+q\right)}{\partial \eta}\right|_{z}+\mathcal{V}\left(C_{v_{c}}+G_{v_{c}}+F_{v_{c}}\right) \\
& \frac{\mathcal{D} w_{c}}{\mathcal{D} t}=-\mathcal{A}_{\sigma} \frac{\partial q}{\partial \sigma}+\mathcal{V}\left(C_{w_{c}}+F_{w_{c}}\right)
\end{aligned}
$$

19 where $\left(\mathcal{A}_{\xi}, \mathcal{A}_{\eta}, \mathcal{A}_{\sigma}\right)=\left(h_{\eta} h_{\sigma}, h_{\xi} h_{\sigma}, h_{\xi} h_{\eta}\right)$ are elementary surfaces and $\mathcal{V}=$ $h_{\xi} h_{\eta} h_{\sigma}$ is the elementary volume. The gravitational term and the vertical 
hydrostatic pressure gradient are absent in (15) because they balance. The two contributions $p^{h}$ and $q$ to the total pressure thus play a completely different role: $p^{h}$ accounts for the gravitational effects whereas $q$ enforces the incompressibility. The additional subscripts $c$ emphasize that the momenta are the Cartesian velocity components. $C_{u_{c}, v_{c}, w_{c}}$ represent the Coríolis force, $G_{u_{c}, v_{c}}$ represent the curvilinear terms arising from the curvature of the grid in the horizontal plane, and $F_{u_{c}, v_{c}, w_{c}}$ represent viscous dissipation, mixing and forcing terms. Note that there is no curvature term in the vertical direction and that $C_{w_{c}}=0$ if the traditional assumption is made. The substantial derivative

$$
\frac{\mathcal{D} *}{\mathcal{D} t}=\frac{\partial}{\partial t}(\mathcal{V} *)+\frac{\partial}{\partial \xi}\left(\mathcal{A}_{\xi} U *\right)+\frac{\partial}{\partial \eta}\left(\mathcal{A}_{\eta} V *\right)+\frac{\partial}{\partial \sigma}\left(\mathcal{A}_{\sigma} W *\right)
$$

uses the flux through the breathing mesh. Note that in the present formalism (16) is not impacted by the new definition of momentum because the flux defined in (3) is identical to the one in Kanarska et al. (2007), up to the area metric factors. Consequently the continuity equation remains identical

$$
\frac{\partial}{\partial t} \mathcal{V}+\frac{\partial}{\partial \xi}\left(\mathcal{A}_{\xi} U\right)+\frac{\partial}{\partial \eta}\left(\mathcal{A}_{\eta} V\right)+\frac{\partial}{\partial \sigma}\left(\mathcal{A}_{\sigma} W\right)=0 .
$$

We can now recast the equations in terms of momentum. First we apply the total derivative (16) to (9)

$$
\begin{aligned}
\frac{\mathcal{D} u}{\mathcal{D} t} & =\frac{\mathcal{D} u_{c}}{\mathcal{D} t}+s_{\xi} \frac{\mathcal{D} w_{c}}{\mathcal{D} t}+w_{c} \frac{\mathcal{D} s_{\xi}}{\mathcal{D} t} \\
\frac{\mathcal{D} v}{\mathcal{D} t} & =\frac{\mathcal{D} v_{c}}{\mathcal{D} t}+s_{\eta} \frac{\mathcal{D} w_{c}}{\mathcal{D} t}+w_{c} \frac{\mathcal{D} s_{\eta}}{\mathcal{D} t} \\
\frac{\mathcal{D} w}{\mathcal{D} t} & =\frac{\mathcal{D} w_{c}}{\mathcal{D} t}
\end{aligned}
$$


237

then substitute the material derivatives of $\left(u_{c}, v_{c}, w_{c}\right)$ with (15) to get

$$
\begin{aligned}
& \frac{\mathcal{D} u}{\mathcal{D} t}=-\left.\mathcal{A}_{\xi} \frac{\partial\left(p^{h}+q\right)}{\partial \xi}\right|_{z}-\mathcal{A}_{\sigma} s_{\xi} \frac{\partial q}{\partial \sigma}+w \frac{\mathcal{D} s_{\xi}}{\mathcal{D} t}+\mathcal{V}\left(C_{u}+G_{u}+F_{u}\right) \\
& \frac{\mathcal{D} v}{\mathcal{D} t}=-\left.\mathcal{A}_{\eta} \frac{\partial\left(p^{h}+q\right)}{\partial \eta}\right|_{z}-\mathcal{A}_{\sigma} s_{\eta} \frac{\partial q}{\partial \sigma}+w \frac{\mathcal{D} s_{\eta}}{\mathcal{D} t}+\mathcal{V}\left(C_{v}+G_{v}+F_{v}\right) \\
& \frac{\mathcal{D} w}{\mathcal{D} t}=-\mathcal{A}_{\sigma} \frac{\partial q}{\partial \sigma}+\mathcal{V}\left(C_{w}+F_{w}\right)
\end{aligned}
$$

where $\left(F_{u}, F_{v}, F_{w}\right)=\left(F_{u_{c}}-s_{\xi} F_{w_{c}}, F_{v_{c}}-s_{\eta} F_{w_{c}}, F_{w_{c}}\right)$ and $\left(G_{u}, G_{v}\right)=\left(G_{u_{c}}, G_{v_{c}}\right)$. The Coriolis terms $C_{u, v, w}$ are detailed in Appendix A. Using the chain rule

$$
\left.\mathcal{A}_{\xi} \frac{\partial *}{\partial \xi}\right|_{z}=\mathcal{A}_{\xi} \frac{\partial *}{\partial \xi}-\mathcal{A}_{\sigma} s_{\xi} \frac{\partial *}{\partial \sigma}
$$

simplifies the expression for the non-hydrostatic pressure gradient

$$
\begin{aligned}
& \frac{\mathcal{D} u}{\mathcal{D} t}=-\left.\mathcal{A}_{\xi} \frac{\partial p^{h}}{\partial \xi}\right|_{z}-\mathcal{A}_{\xi} \frac{\partial q}{\partial \xi}+w \frac{\mathcal{D} s_{\xi}}{\mathcal{D} t}+\mathcal{V}\left(C_{u}+G_{u}+F_{u}\right) \\
& \frac{\mathcal{D} v}{\mathcal{D} t}=-\left.\mathcal{A}_{\eta} \frac{\partial p^{h}}{\partial \eta}\right|_{z}-\mathcal{A}_{\eta} \frac{\partial q}{\partial \eta}+w \frac{\mathcal{D} s_{\eta}}{\mathcal{D} t}+\mathcal{V}\left(C_{v}+G_{v}+F_{v}\right) \\
& \frac{\mathcal{D} w}{\mathcal{D} t}=-\mathcal{A}_{\sigma} \frac{\partial q}{\partial \sigma}+\mathcal{V}\left(C_{w}+F_{w}\right) .
\end{aligned}
$$

(21) is the new system of momentum equations we propose to use to integrate the primitive equations in sigma-coordinates model when the hydrostatic assumption is relaxed. The terms in (21) involving the substantial derivatives of $s_{\xi}$ and $s_{\eta}$ can also be written as

$$
\begin{aligned}
& w \frac{\mathcal{D} s_{\xi}}{\mathcal{D} t}=w \mathcal{V} \frac{\partial s_{\xi}}{\partial t}+w U \mathcal{A}_{\xi} \frac{\partial s_{\xi}}{\partial \xi}+w V \mathcal{A}_{\eta} \frac{\partial s_{\xi}}{\partial \eta}+w W \mathcal{A}_{\sigma} \frac{\partial s_{\xi}}{\partial \sigma} \\
& w \frac{\mathcal{D} s_{\eta}}{\mathcal{D} t}=w \mathcal{V} \frac{\partial s_{\eta}}{\partial t}+w U \mathcal{A}_{\xi} \frac{\partial s_{\eta}}{\partial \xi}+w V \mathcal{A}_{\eta} \frac{\partial s_{\eta}}{\partial \eta}+w W \mathcal{A}_{\sigma} \frac{\partial s_{\eta}}{\partial \sigma}
\end{aligned}
$$

245 This derivation makes (22) inherently new terms compared to Kanarska et al. 246 (2007). The system (21) can be derived independently by application of 


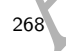

Hamilton's principle (Appendix C). It yields the vector-invariant form. After a bit of algebra the flux form can be recovered along with the expression for the curvilinear terms. This alternative derivation blends the contributions of (22) and $\mathcal{V} G_{u, v}$ into a same term. Finally note that the chain rule (20) can also be applied to the hydrostatic pressure gradient term in (21). This introduces a buoyancy term in the horizontal components of the momentum equations, like in the variational approach (Appendix C). In practice the hydrostatic pressure gradient and this buoyancy termare computed together to limit the pressure gradient error (Shchepetkin and McWilliams, 2003).

Finally we want to emphasize that there is no necessity to prognose the covariant components. A perfectly valid choice could be to prognose the contravariant ones $\left(U, V, W^{a}\right)$. In fact, it seems that the good choice of prognostic variables depend on the formulation of the momentum equations: in vector-invariant, the covariant components appear to be natural (see Eq C.4), in flux-form, the contravariant components are more natural.

\section{Discrete operators for the non-hydrostatic pressure}

In this section we first show how the Poisson equation for pressure can be derived from the definitions of the kinetic energy and the divergence operator. We then discretize the kinetic energy and deduce the discretizations for the T matrix and the Poisson equation.

3.1. Pressure projection in matrix formulation

Following Kanarska et al. (2007), we will use the language of pressure projection methods (Chorin, 1968; Bell et al., 1989). These methods refer to a class of fractional step, implicit algorithms for unsteady flows that satisfy a 
non-divergence constraint. In the fractional step method a provisional velocity field is constructed first by advancing the momentum equations in time without enforcing the no-divergence constraint. Subsequently, the divergent part of velocity field is removed by a projection to the no-divergence subspace. Here, we will present a derivation following Molemaker et al. (2005) to arrive, first at an expression for the operators in matrix form and second, specifically in terms of discrete coefficients.

We define the diagonal matrices $\mathbf{L}, \mathbf{A}, \mathbf{V}$ corresponding to grid lengths, cell face areas and cell volumes working on velocity points. The elements of $\mathbf{L}$ are the Lamé coefficients. The matrices are related by $\mathbf{V}=\mathbf{A L}$. We define $\mathbf{d}$ the differentiation matrix acting on cell centers (rho-points) and returning gradient components at face centers (velocity points). $\mathbf{d}$ carries no dimension. The divergence is $\mathbf{D}=-\mathbf{d}^{\mathbf{T}} \mathbf{A}$. Note the minus and the transpose. The divergence works on fluxes and returns finite volume scalars (scalar times the elementary volume).

Using (12), we can write the relation between fluxes and momenta compactly in matrix form as:

$$
X^{a}=\mathbf{T} x
$$

where $x=(\mu, v, w)^{T}$ is the solution vector in terms of momenta and $X^{a}=$ $\left(U, V, W^{a}\right)^{T}$ the equivalent in terms of fluxes. We use the domain integrated kinetic energy $\mathcal{K}=\frac{1}{2} x^{T} \mathbf{V} X^{a}$ to define the inner product of two momenta $x$ and $y$

$$
<x, y>=x^{T} \mathbf{V T} y
$$

${ }_{292}$ To be an inner product VT needs to be symmetric. This is achieved by 
293 discretizing the kinetic energy in such way that

$$
\mathcal{K}=\frac{1}{2}(\mathbf{L} x)^{T} \tilde{\mathbf{T}}(\mathbf{L} x),
$$

${ }_{294}$ where $\tilde{\mathbf{T}}$ is a symmetric matrix. The discretization of $\mathbf{T}$ is then deduced

$$
\mathbf{T}=\mathbf{A}^{-1} \tilde{\mathbf{T}} \mathbf{L} .
$$

295 The non-divergence condition is a linear constraint of the flow that, using 296 (17), can now be written in terms of the momenta as:

$$
\mathrm{DT} x_{D}=0
$$

297 where the subscript $D$ indicates a solution vector that satisfies the no-divergence

The correction is of the form

$$
\epsilon=\mathbf{V}^{-1} \mathbf{D}^{T} p
$$

${ }_{306}$ with $p$ a scalar field, because

$$
<x_{D}, \mathbf{V}^{-1} \mathbf{D}^{T} p>=x_{D}^{T} \mathbf{T}^{T} \mathbf{D}^{T} p=\left(\mathbf{D} \mathbf{T} x_{D}\right)^{T} p=0 .
$$


$p$ acts as a Lagrange multiplier. Its function is to help satisfy the linear constraint of non-divergence. Physically speaking, the pressure functions as such for an incompressible flow, and we see that the correction vector $\epsilon$ is, in fact, the pressure gradient. We now have the following system:

$$
\begin{array}{r}
\mathbf{V T} x-\mathbf{V T} x_{D}=(\mathbf{D T})^{T} p \\
\mathbf{D T} x_{D}=0 .
\end{array}
$$

\subsection{Variable staggering}

318

We describe here how the momentum and flux can be discretized in such way that the operators grad and div operating on them acquire the simplest 

343 this below.

Scalar quantities are naturally discretized at either cell centers or cell 


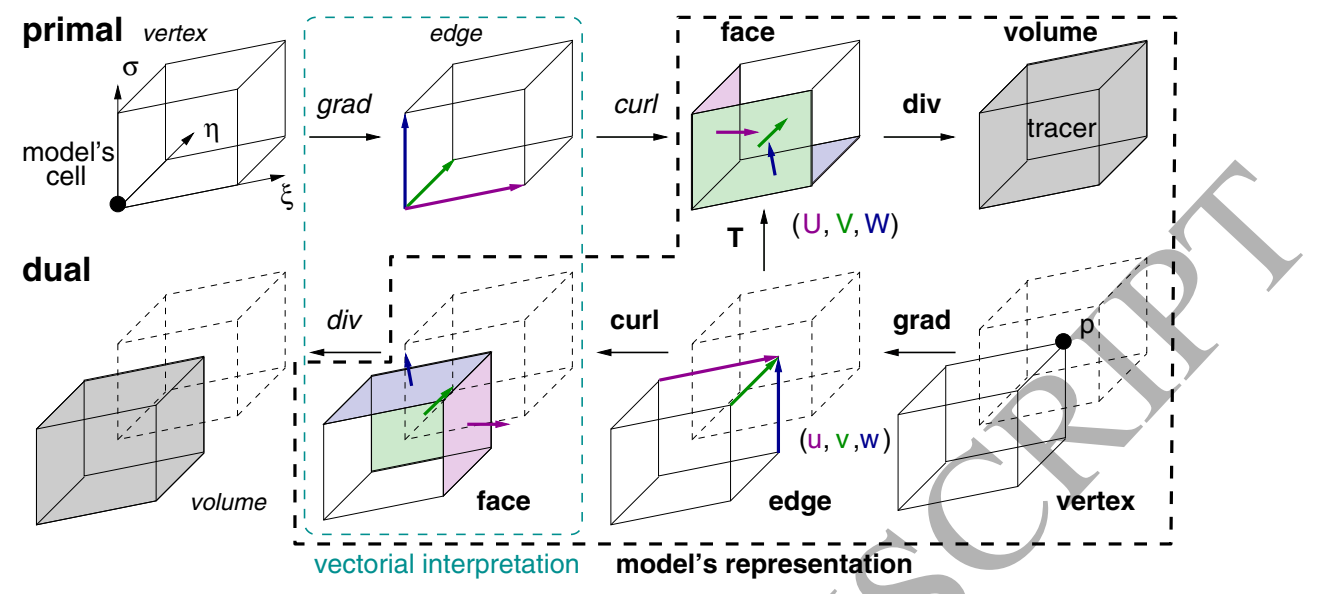

Figure 1: Staggering of variables on the primal and dual grids according to their nature: velocity, pressure or tracer. Contravariant components are defined on the primal, covariant components on the dual. They are related by the $\mathbf{T}$ transformation. By interpreting the contravariant as flux, living on faces, and the covariant as gradient terms, living on the edges, this makes the pressure gradient operation and the divergence of the flux very simple. This interpretation is consistent with a discretization of the pressure at the primal cell centers (dual vertices) and a finite volúme interpretation of tracers (primal volume). Each object is connected to its right-neighbour (primal) and left-neighbour (dual) by a differentiation operator which, depending on the object, is the gradient, the curl or the divergence. The vectorial interpretation is illustrated by the cyan dashed box. The model's objects and operators (bold) are delimited by the black dashed box.

volumes depending on whether the quantity is a true scalar, like pressure, or an integrated quantity, like tracer content. Vectors are naturally discretized at either cell edges or cell faces. Contravariant components are defined on the primal whereas the covariant components are on the dual. The two interpretations for the contravariant components can now be enriched by this geometric framework: the contravariant components are either i) on the faces of the primal or ii) on the edges of the primal, which is the vectorial inter- 

${ }_{375}$ when they differ from $i, j$ or $k$, e.g. $\phi_{i-1}$ will stand for $\phi_{i-1, j, k}$. There is 376

pretation. Interpretation i) is the one we made, it makes the flux naturally transformed into a volume via the divergence operator. With interpretation ii), the divergence of the contravariant components is not a trivial operation. Similarly, the momentum should behave like a gradient, because it is covariant. It can be discretized either i) on the edges of the dual or on ii) the faces. To be consistent, it has to be one edges. It makes the computation of the pressure gradient a simple finite difference. By using discretization ii), the expression of the pressure gradient acting on the momentum would be complicated.

The consistency of i) with the C-grid discretization practice is even deeper: the finite volume discretization of tracers implies that the flux sits at the primal faces, and the discretization of pressure at cell centers implies that the covariant velocity sits at the dual edges. Finally, having the contravariant components on the primal faces makes the operation $\operatorname{div} \mathbf{U}$ covariant, i.e. coordinates independent.

The $\mathbf{T}$ matrix transforms objects from the dual edges to the primal faces. Lastly, from figure 1 it is clear that the vorticity, defined as the curl of the momentum lives at the dual faces. It inherits covariant components. The vorticity appears explicitly in the vector-invariant form. It is only a diagnostic quantity in flux-form.

From now on, each variable is indexed with a triplet of indices $(i, j, k)$. Faces and edges with index $(i, j, k)$ are located to the west, south and bottom of the cell $(i, j, k)$. To lighten the notation the indices will be indicated only $n_{z}$ cells in the vertical and $n_{z}+1 w$-levels. We define the right difference 
operator $\delta_{i, r}$ acting on the field $\phi_{i, j, k}$ in the direction $\xi$ as $\delta_{i, r}[\phi]=\phi_{i+1}-\phi$ and the left difference operator $\delta_{i, l}[\phi]=\phi-\phi_{i-1}$. We define the right averaging operator $\bar{\phi}^{i, r}=\left(\phi_{i+1}+\phi\right) / 2$ and the left averaging one $\bar{\phi}^{i, l}=\left(\phi+\phi_{i-1}\right) / 2$. Likewise we define the same operators acting in direction $\eta$ with index $j$, and in direction $s$ with index $k$.

Grid spacing $\left(h_{\xi}, h_{\eta}, h_{\sigma}\right)$, and local slopes $\left(s_{\xi}, s_{\eta}\right)$ are also defined at cell centers with

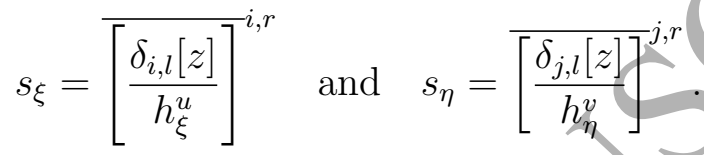

A superscript of $u, v, w$ denotes a quantity that has been averaged to the position of its respective velocity, e.g. $h_{\xi}^{u}=\delta_{i, r}\left[h_{\xi}\right]$. In particular $h_{\sigma}^{w}$ is only half of its value at the bottom $(k=1)$ and top level $\left(k=n_{z}+1\right)$. Faces areas are defined at velocity points using product of two lengths, e.g. $\mathcal{A}_{\xi}^{u}=h_{\eta}^{u} h_{\sigma}^{u}$. Volumes are defined at velocity points using the product of the lengths in each direction, e.g. $\mathcal{V}^{u}=h_{\xi}^{u} h_{\eta}^{u} h_{\sigma}^{u}$.

\subsection{Discretization of the gradient and divergence}

As announced, the discretization of the gradient and divergence boil down to simple finite differences. If we denote $X=(U, V, W)^{T}$ the flux vector and $\delta=\mathbf{D} X$ the result of the action of $\mathbf{D}$, then the discretization of the divergence reads

$$
\delta_{i, r}\left[\mathcal{A}_{\xi}^{u} U\right]+\delta_{j, r}\left[\mathcal{A}_{\eta}^{v} V\right]+\delta_{k, r}\left[\mathcal{A}_{\sigma}^{w} W\right]=\delta
$$

The definition is valid at all grid points, including at the boundaries. The bottom boundary condition is handled outside of the divergence by simply 397 setting $W=0$. The gradient is defined as the skew-adjoint of $\mathbf{D}$. For interior 
398

points it is easy to show that

$$
h_{i}^{u} u_{i}=\delta_{i, l}[q], \quad h_{i}^{v} v_{i}=\delta_{j, l}[q], \quad h_{i}^{w} w_{i}=\delta_{k, l}[q],
$$

where $q$ is a scalar defined on the dual vertices and $(u, v, w)$ are the (coyariant) gradient components. The expression for the curl is given in Appendix C.

It is basically computed by estimating the circulation around each face.

\subsection{Discretization of the kinetic energy}

As said above, the discretization of the $\mathbf{T}$ matrix derives from $\mathcal{K}$ written in the form (25). To discretize $\mathcal{K}$ we start from the continuous expression

$$
\mathcal{K}=\iiint\left[\frac{1}{2}\left(u^{2}+v^{2}+\gamma w^{2}\right)-\left(s_{\xi} u w+s_{\eta} v w\right)\right] \mathcal{V} d \xi d \eta d \sigma
$$

with $\gamma=1+s_{\xi}^{2}+s_{\eta}^{2}$, and split it into several contributions

$$
\mathcal{K}=\mathcal{K}_{\text {int }}+\mathcal{K}_{\text {cross }}+\mathcal{K}_{\text {bottom }}
$$

where $\mathcal{K}_{\text {bottom }}$ accounts for all terms involving the bottom vertical momentum $w_{b}(w$ at $k=1), \mathcal{K}_{\text {cross }}$ accounts for the cross terms $u w$ and $v w$, and $\mathcal{K}_{\text {int }}$ is the remainder. The interior kinetic energy

$$
\mathcal{K}_{i n t}=\frac{1}{2} \sum_{i, j}\left\{\sum_{k=1}^{n_{z}} u^{2} \mathcal{V}^{u}+\sum_{k=1}^{n_{z}} v^{2} \mathcal{V}^{v}+\sum_{k=2}^{n_{z}+1} \gamma^{w} w^{2} \mathcal{V}^{w}\right\}
$$

is the simplest term. Each component is weighted with its volume defined at the component location. This part of the kinetic energy encodes the diagonal terms of the $\tilde{\mathbf{T}}$ matrix, which are $\left(A^{u} / h_{\xi}^{u}, A^{v} / h_{\eta}^{v}, c^{w} A^{w} / h_{\sigma}^{w}\right)$. The cross terms 
contribution reads

$$
\begin{aligned}
& \left.\left.\mathcal{K}_{\text {cross }}=-\frac{1}{2} \sum_{i, j}\left[s_{\xi} h_{\eta} \overline{\left[h_{\xi}^{u} u\right.}\right]^{i, r}+s_{\eta} h_{\xi} \overline{\left[h_{\eta}^{v} v\right]}\right]^{j, r}\right]_{k=1}\left[h_{\sigma}^{w} w\right]_{k=2} \\
& -\sum_{i, j} \sum_{k=2}^{n_{z}-1}\left[s_{\xi} h_{\eta}{\overline{\left[h_{\xi}^{u} u\right]}}^{i, r}+s_{\eta} h_{\xi}{\overline{\left.h h_{\eta}^{v} v\right]}}^{j, r}\right]{\overline{\left[h_{\sigma}^{w} w\right]}}^{w, r} \\
& -\frac{1}{2} \sum_{i, j}\left[s_{\xi} h_{\eta} \overline{\left[h_{\xi}^{u} u\right]^{i, r}}+s_{\eta} h_{\xi} \overline{\left[h_{\eta}^{v} v\right]^{j, r}}\right]_{k=n z}\left[h_{\sigma}^{w} w\right]_{k=n z}
\end{aligned}
$$

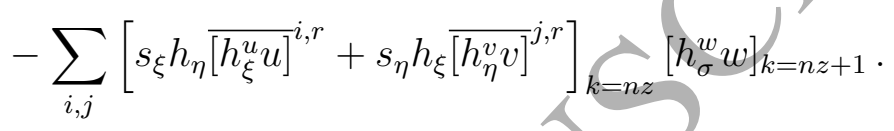

All terms are defined at cell centers (rho points). The yolume elements have been carefully split into three length elements to form elementary circulations to ensure (25). The choice to average the terms at cell centers allows for the maximum of terms cancellation in the resulting pressure matrix. The sum has been split in horizontal slabs: in the bottom level $(k=1)$ we have removed the part involving $w_{b}$, in the top level $\left(k=n_{z}\right)$ we have introduced an extra factor 2 to compensate for $h_{\sigma}^{w}$ being halved.

The bottom contribution accounts for the kinetic energy density $K=$ $\frac{1}{2} \gamma w^{2}-\left(s_{\xi} u w+s_{\eta} v w\right)$ integrated over the bottom cells lower half volume. The $u^{2}$ and $v^{2}$ are already accounted in $\mathcal{K}_{i n t}$. Because $w$ at the bottom is not a prognostic variable, it should be substituted with its diagnostic relation $\gamma w=s_{\xi} u+s_{\eta} v$, obtained from (11), the definition of $W^{a}$, and the fact that the breathing vanishes at the bottom boundary $\left(\partial_{t} z=0\right)$. After substitution, the kinetic energy density reads $K=-\frac{1}{2 \gamma}\left[\left(s_{\xi} u\right)^{2}+\left(s_{\eta} v\right)^{2}+2 s_{\xi} s_{\eta} u v\right]$, which is the continuous density we use for the discretization at the bottom. The 
discretized volume-integrated bottom kinetic energy then reads

$$
\mathcal{K}_{\text {bottom }}=-\frac{1}{4} \sum_{i, j, k=1}\left\{{\overline{\left[\frac{s_{\xi}^{2}}{\gamma}\right]}}^{i, l} u^{2} \mathcal{V}^{u}+{\overline{\left[\frac{s_{\eta}^{2}}{\gamma}\right]}}^{j, l} v^{2} \mathcal{V}^{v}+2 \frac{s_{\xi} s_{\eta} h_{\sigma}}{\gamma}\left[\overline{\left.h_{\xi}^{u} u\right]^{i, r}} \overline{\left[h_{\eta}^{v} v\right]} j,\right\}\right.
$$

$$
U=\left(1-{\overline{\left[\frac{s_{\xi}^{2}}{2 \gamma}\right.}}^{i, l}\right) u-\frac{1}{2 \mathcal{A}_{\xi}^{u}}\left({\overline{\frac{s_{\xi} s_{\eta} h_{\sigma}}{\gamma}{\overline{\left[h_{\eta}^{v} v\right]}}^{j, r}}}^{i, l}+{\overline{{\frac{s \xi}{\xi} h_{\eta}}_{\sigma}}\left[h_{\sigma}^{w} w\right]_{k=2}}^{i, l}\right)
$$

\section{We have for the horizontal flux $U$}

- In the interior $\left(1<k<n_{z}\right)$

$$
U=u-\frac{1}{\mathcal{A}_{\xi}^{u}}{\overline{s_{\xi}}}_{h_{\eta}{\overline{\left[h_{\sigma}^{w} w\right]}}^{w, r}, i l}^{i}
$$

- At the bottom $k=1$ 

${ }_{441}$ condition number is controlled by the grid characteristics: grid steps and ${ }_{442}$ number of grid points. 


\section{4. Discussion}

${ }_{465}$ We now assess the properties of the discretization on a test-case. The 466 ergy fully determine the boundary conditions on the pressure equation. At the surface the boundary condition is of Dirichlet type $(p=0)$ whereas at the bottom and laterally it is of Neumann type. To exhibit them we use the property that the flux is divergent-free $<p, \mathbf{D T} x_{D}>=0$, then make use of the adjoint to find $<\mathbf{D}^{T} p, \mathbf{T} x_{D}>=0$ which expresses that the pressure force does not work. By expanding this inner product as a sum on velocity points we recover the discrete kinetic energy for which one of the momentum component is replaced with the pressure gradient. Tn particular the $w^{2}$ term at $\left(i, j, n_{z}+1\right)$ in $\mathcal{K}_{i n t}$ is now $-\gamma^{w} \mathcal{V}^{w} w p_{i, j, n} / h_{i, j, n_{z+1}}^{w}$, from which we deduce the vertical pressure gradient at the surface is $\partial_{z} p=-p_{i, j, n} / h_{i, j, n_{z}+1}^{w}$. This discretization is indeed a vertical gradient if $p_{i, j, n_{z}+1 / 2}=0$, thus proving that the surface boundary condition is of Dirichlet type. The surface boundary condition is realized in the Poisson stencil by the $A_{\sigma}^{w} / h_{\sigma}^{w}$ term at $k=n_{z}+1$ in the diagonal coefficient $P_{1}($ see (B.2) in Appendix B). The bottom boundary condition is handled by $\mathcal{K}_{\text {bot }}$ and behaves such that $W=0$ at the bottom. The pressure gradient at the bottom is not defined but its associated vertical flux $\left(\mathbf{T D}^{T} p\right)$ is zero there, which corresponds to a Neumann boundary condition. The lateral boundary conditions is treated by assuming the covariant velocity, either $u$ or $v$, vanishes. This removes in the Poisson matrix all connections between interior and outer pressure points.

al is test the discretized projection in a configuration simple enough, yet

The combination of the discrete divergence and the discrete kinetic en- 
ensuring that none of the 15 coefficients of the Poisson matrix vanishes. The comparison with an analytical solution would have been ideal but we have found none with a genuinely three-dimensional topography.

The test-case, expressed in Cartesian coordinates, consists in projecting a divergent velocity field $(u, v, w)=(0,0, \cos (\pi x / L))$ onto the divergent-free subspace in the closed domain $(x, y, z) \in[0, L] \times[0, L] \times[-H+h(x, y), 0]$, where $h(x, y)=h_{0} \exp \left(-\alpha(x-L / 2)^{2}-\alpha(y-L / 2)^{2}\right)$ is a sea-mount topography. The numerical values are $L=10 \mathrm{~km}, H=4 \mathrm{~km}, h_{0}=2 \mathrm{~km}, \alpha=25 / L^{2}$. The test-case is particularly severe for the bottom boundary condition because the initial flow field is already divergence-free but it is far from being the solution because it does not satisfy the no-flow bottom boundary condition. The right hand side for the Poisson equation is thus zero everywhere except along the topography.

Even though the test-case is relatively simple, it has no analytical solution. It is therefore difficult to prove that the numerical solution obtained with the 15-point stencil is correct. To circumvent this issue we have computed the solution using two other methods: the tall 25-point stencil obtained with Cartesian components and the 7 points stencil derived using a z-level discretization. The 25-point stencil matrix can be written as $\mathbf{P}_{w}=\mathbf{D}_{w} \mathbf{V}^{-1} \mathbf{D}_{w}^{T}$, where the divergence operator $\mathbf{D}_{w}$ has a wider stencil than $\mathbf{D}$ because of the extra derivatives coming from the chain rule. The approach closely follows Aluclair et al. (2011) but it differs by explicitly obtaining a symmetric matrix. In the z-levels discretization, the topography is handled via a mask array that flags cells outside the domain. The resulting matrix is similar to what is implemented in MITgcm (Marshall et al., 1997). In both cases, 
the bottom boundary condition is trivially satisfied by resorting a divergence matrix that does not uses the $w$ of the bottom level to compute the divergence in the first level. In addition to these three matrices we also test the impact of the extra-care needed to handle to bottom boundary condition in the 15-point stencil case by implementing a degraded version of it in which we simply remove in the $\mathbf{T}$ matrix all the contributions from the $\mathcal{K}_{\text {bot }}$ term. This matrix is obtained by setting the flag $\mu=0$ in the Poisson stencil given in appendix.

We solve the problem on a series of grids $\left(n_{x}, n_{y}, n_{z}\right)=2^{n} \times(25,25,10)$ with doubling resolution. The matrices are implemented in Matlab. Because the grids need to fit in RAM of a single chipset, we were able to test the range $n=0,1,2,3$ for the four matrices. The solution is a non trivial flow. Figure 2 shows the Cartesian $u_{c}$ and $w_{c}$ components of the flow in the mid-plane $y=L / 2$ for $n=3$. The flow has on one side a down-welling branch emanating from the surface and an upwelling branch on the other. Inbetween, the flow is passing around (not shown) and over the sea-mount. The four implementations give an identical flow. At this resolution the differences are several orders of magnitude smaller than the solution. To quantitatively measure the convergence we compute the kinetic energy $\mathcal{K}$ of each solution. Results are shown in Figure 3. They clearly converge to the same value $\mathcal{K}_{\infty}$, indicated by the tick black line, albeit with a different convergence rate. The 15 - and the 25-point stencils converge more rapidly to $\mathcal{K}_{\infty}$. Specifically their increment of accuracy $\delta \mathcal{K}=\mathcal{K}(n)-\mathcal{K}(n-1)$ decreases by a factor of 4 when the resolution is doubled, implying that $\mathcal{K}$ is of the form $\mathcal{K}=\mathcal{K}_{\infty}+c 4^{-n}$, where $c$ is a constant. We can then use the Richardson extrapolation on the 
15 -point results to estimate this limit value as $\mathcal{K}_{\infty} \approx \mathcal{K}(n)+\delta \mathcal{K}(n) / 3$.

This allows to monitor the absolute error $\left|\mathcal{K}-\mathcal{K}_{\infty}\right|$ (inset of Fig. 3). This figure confirms that the 15 - and 25-point stencil converge to the solution at second order, as indicated by the -2 slope. Interestingly, the 15-point stencil is slightly more accurate than the 25 -point stencil. This can be traced back to slightly smoother vertical shears near the bottom. The z-levels only converges at first order, which is consistent with the discretization of the topography that is only first order. For that case the solution has the footprint of steps near the topography (not shown). The degraded 15-point stencil also has a global order 1 convergence. Comparisons with the full 15-point stencil reveal that the horizontal flux vertical profile is discontinuous at the bottom level, generating a maximum of error there. Because the problem is essentially global this impacts the solution everywhere. The contribution of the horizontal cross terms in the bottom level ensures a uniform second order estimate of the velocities everywhere.

\section{Conclusion}

We have presented an alternative system of equations for the primitive equations written in sigma coordinates with a breathing mesh. It is based on the recognition of two sets of components for the velocity: a contravariant set, involved in the flux definitions, and a covariant one, involved in the momentum definition. The two sets are related by a linear transformation, the metric tensor, fully determined by the kinetic energy. We provide the momentum equations written for the covariant components in both vectorinvariant and flux-form. We show that within the hydrostatic assumption, 

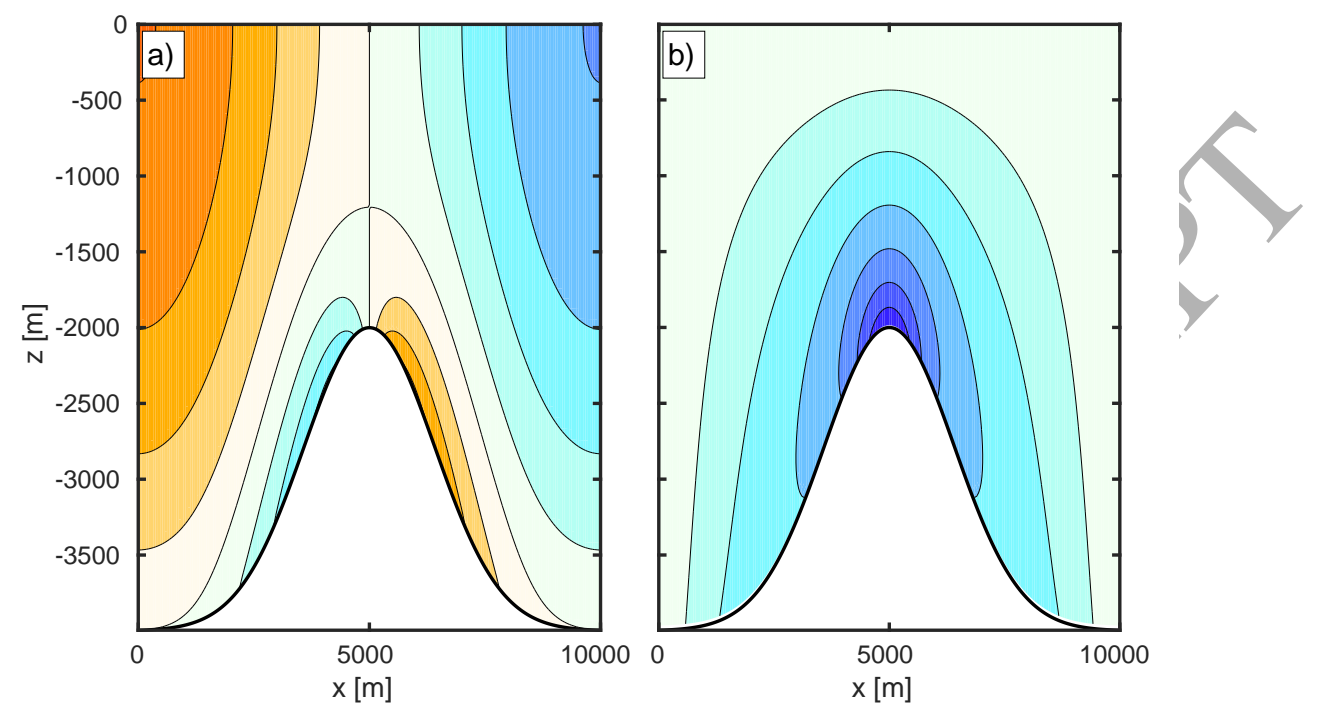

Figure 2: Vertical section of the velocity Cartesian components $w_{c}$ (a) and $u_{c}$ (b) at $y=L / 2$, for the full solution obtained with the 15 -point stencil matrix.

548 ${ }_{549}$ can be implemented numerically yielding to a compact symmetric stencil for 550 the Poisson matrix with only 15 points. The discretization is done at the level 


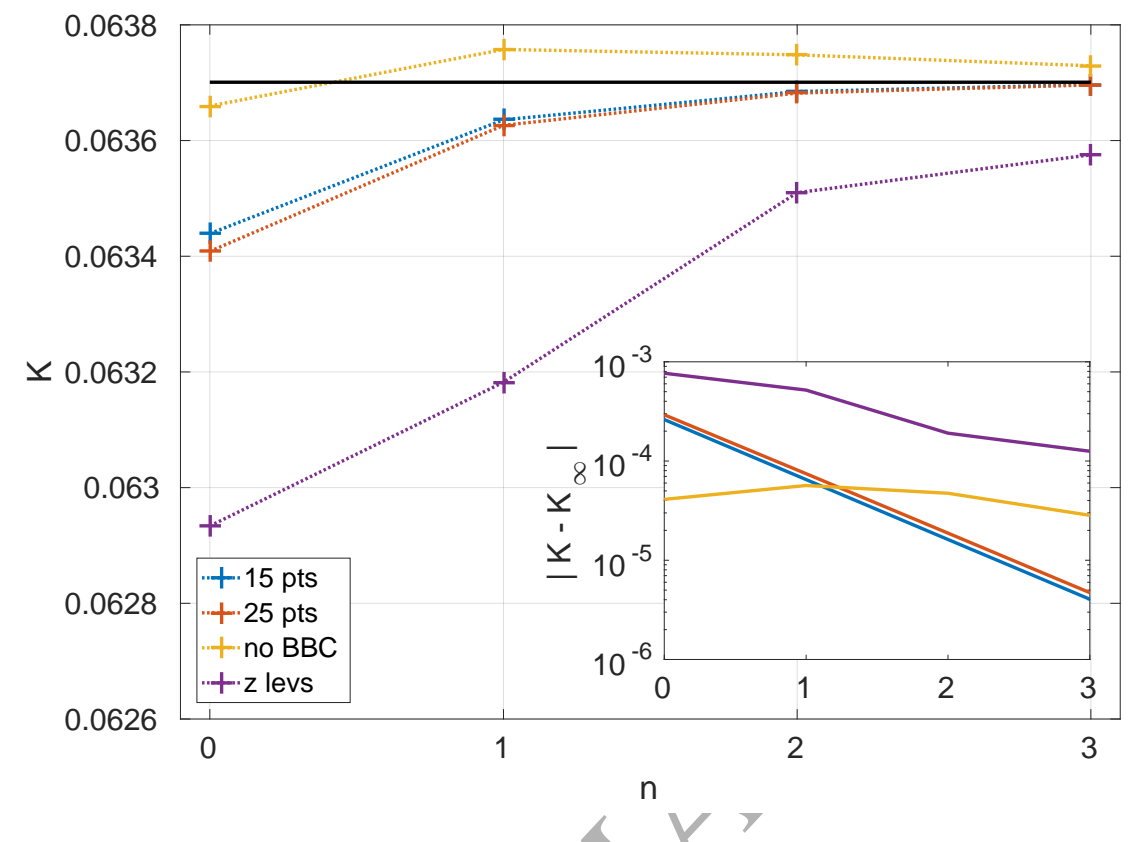

Figure 3: Kinetic energy as a function of resolution $n$ for four different Poisson matrices: the 15-point stencil $\mathbf{P}$ matrix (blue), the 25 points Cartesian matrix $\mathbf{P}_{w}$ (red), the 15-point matrix with the bottom boundary condition removed (yellow), the 7 points z-levels matrix (purple). The thick line is the limit value $\mathcal{K}_{\infty}$ obtained by Richardson extrapolation. The inset shows the absolute error $\left|\mathcal{K}-\mathcal{K}_{\infty}\right|$ as a function of resolution.

of the kinetic energy, which ensures that the pressure work vanishes globally. It also ensurès the symmetry of the stencil. We show how the no-flow bottom boundary condition can be discretized with a second order accuracy. This discretization is also done directly at the level of the kinetic energy.

This approach opens a promising way of solving NH physics in a sigma coordinates model. The full implementation of this system of equations in ${ }_{557}$ an ocean model will be reported in a forthcoming paper. 
558

559

Rearranging using (3) and (9) again, then defining

$$
f_{\sigma}=f_{z}-s_{\xi} f_{\xi}-s_{\eta} f_{\eta},
$$

566

yields)

\section{Appendix A. Coriolis terms} components of the Earth angular speed $\Omega$, namely

$$
\begin{aligned}
& f_{\xi}=2 \Omega \cos \phi \sin \alpha \\
& f_{\eta}=2 \Omega \cos \phi \cos \alpha \\
& f_{z}=2 \Omega \sin \phi,
\end{aligned}
$$
Cartesian velocity components are

$$
\begin{aligned}
& C_{u_{c}}=-v_{c} f_{z}+w_{c} f_{\eta} \\
& C_{v_{c}}=+u_{c} f_{z}-w_{c} f_{\xi} \\
& C_{w_{c}}=-u_{c} f_{\eta}+v_{c} f_{\xi} .
\end{aligned}
$$

$$
\begin{aligned}
C_{u} & =C_{u_{c}}+s_{\xi} C_{w_{c}} \\
C_{v} & =C_{v_{c}}+s_{\eta} C_{w_{c}} \\
C_{w} & =C_{w_{c}} .
\end{aligned}
$$

In the non-traditional approximation the Coriolis terms involve the three

where $\alpha$ is the angle between the $\xi$ and the zonal direction and $\phi$ is the latitude. From Appendix B of Kanarska et al. (2007) the Coriolis terms for

Yr.

$$
\begin{aligned}
& C_{u}=-V f_{\sigma}+W^{a} f_{\eta} \\
& C_{v}=+U f_{\sigma}-W^{a} f_{\xi} \\
& C_{w}=-U f_{\eta}+V f_{\xi} .
\end{aligned}
$$



${ }_{580}$ and $\mu=0$ for the degraded 15-point matrix in which the bottom boundary

The discretization of the Poisson matrix follows from (35). Its action on a discrete scalar field $q_{i, j, k}$ can be written as

$$
\begin{array}{r}
P_{1} q+P_{2} q_{k-1}+P_{2, k+1} q_{k+1}+P_{3} q_{j-1, k+1}+P_{3, j+1, k-1} q_{j+1, k-1} \\
+P_{4} q_{j-1}+P_{4, j+1} q_{j+1}+P_{5} q_{j-1, k-1}+P_{5, j+1, k+1} q_{j+1, k+1} \\
+P_{6} q_{i-1, k+1}+P_{6, i+1, k-1} q_{i+1, k-1}+P_{7} q_{i-1}+P_{7, i+1} q_{i+1} \\
+P_{8} q_{i-1, k-1}+P_{8, i+1, k+1} q_{i+1, k+1}+P_{9} q_{i-1, j-1}+P_{9, i+1, j+1} q_{i+1, j+1} \\
+P_{10} q_{i-1, j+1}+P_{10, i+1, j-1} q_{i+1, j-1}=r_{i, j, k}
\end{array}
$$

where the coefficients $P_{l}, l=1,2, \ldots 10$, define the stencil of the matrix and $r_{i, j, k}$ is the result. Coefficients pointing to indices outside of the index range are set to 0 . To lighten the notation the indices are indicated only when they differ from $i, j$ or $k$, e.g. $P_{2}$ stands for $P_{2, i, j, k}$. Each coefficient has a general expression for interior levels $1<k<n_{z}$ but differ at the top and bottom levels. We define the indicatrix function $\Phi_{l ; i, j, k}$ that is 0 for $k \neq l$ and 1 for $k=l$. We also define a flag $\mu=1$ for the full 15-point Poisson matrix 
581

$$
P_{1}=\frac{A_{\sigma}^{w}}{h_{\sigma}^{w}}\left(1-\Phi_{1}\right)+\left.\frac{A_{\sigma}^{w}}{h_{\sigma}^{w}}\right|_{k+1}+\frac{A_{\eta}^{v}}{h_{\eta}^{v}}+\left.\frac{A_{\eta}^{v}}{h_{\eta}^{v}}\right|_{j+1}+\frac{A_{\xi}^{u}}{h_{\xi}^{u}}+\left.\frac{A_{\xi}^{u}}{h_{\xi}^{u}}\right|_{i+1} .
$$

Recall that $A_{\sigma}^{w} / h_{\sigma}^{w}$ is well defined at $k=n_{z}+1$. The other coefficients read

$$
\begin{aligned}
P_{2} & =\frac{A_{\sigma}^{w}}{h_{\sigma}^{w}} \gamma^{w}\left(1-\Phi_{1}\right) \\
P_{3} & =\frac{1}{4}\left(s_{\eta} h_{\xi}+s_{\eta, j-1} h_{\xi, j-1}\right)\left(1-\Phi_{n_{z}}\right) \\
P_{4} & =\frac{A_{\eta}^{v}}{h_{\eta}^{v}}\left(1-\frac{1}{4}\left[\frac{s_{\eta}^{2}}{\gamma}+\frac{s_{\eta, j-1}^{2}}{\gamma_{j-1}}\right] \Phi_{1} \mu\right)+\frac{1}{4}\left(s_{\eta} h_{\xi}-s_{\eta, j-1} h_{\xi, j-1}\right)\left(\Phi_{n z}+\Phi_{1}\right) \\
P_{5} & =-\frac{1}{4}\left(s_{\eta} h_{\xi}+s_{\eta, j-1} h_{\xi, j-1}\right)\left(1-\Phi_{1}\right) \\
P_{6} & =\frac{1}{4}\left(s_{\xi} h_{\eta}+s_{\xi, i-1} h_{\eta, i-1}\right)\left(1-\Phi_{n_{z}}\right) \\
P_{7} & =\frac{A_{\xi}^{u}}{h_{\xi}^{u}}\left(1-\frac{1}{4}\left[\frac{s_{\xi}^{2}}{\gamma}+\frac{s_{\xi, i-1}^{2}}{\gamma_{i-1}}\right] \Phi_{1} \mu\right)+\frac{1}{4}\left(s_{\xi} h_{\eta}-s_{\xi, i-1} h_{\eta, i-1}\right)\left(\Phi_{n z}+\Phi_{1}\right) \\
P_{8} & =-\frac{1}{4}\left(s_{\xi} h_{\eta}+s_{\xi, i-1} h_{\eta, i-1}\right)\left(1-\Phi_{1}\right) \\
P_{9} & =-\frac{1}{8}\left(\frac{s_{\xi, i-1} s_{\eta, i-1} h_{\sigma, i-1}}{\gamma_{i-1}}+\frac{s_{\xi, j-1} s_{\eta, j-1} h_{\sigma, j-1}}{\gamma_{j-1}}\right) \Phi_{1} \mu \\
P_{10} & =\frac{1}{8}\left(\frac{s_{\xi, i-1} s_{\eta, i-1} h_{\sigma, i-1}}{\gamma_{i-1}}+\frac{s_{\xi, j+1} s_{\eta, j+1} h_{\sigma, j+1}}{\gamma_{j+1}}\right) \Phi_{1} \mu
\end{aligned}
$$

As it is seen, only 8 coefficients are needed away from the top and bottom boundaries. This is a manifestation of the both the compactness and the symmetry of the stencil. 


\section{Appendix C. Variational derivation}

In this section we recover the momentum equations (21) with a variational derivation using Hamilton's principle. However we were able to do it only for the case of a non-breathing grid, so that all terms involving $\partial_{t} z$, $\partial_{t} s_{\xi}$ and $\partial_{t} s_{\eta}$ vanish. We also remove the full Coriolis force because the yariational approach requires to have horizontal Lamé coefficients $\left(h_{\xi}, h_{\eta}\right)$ that vary in the vertical, which corresponds to the deep atmosphere assumption in atmospheric models (Tort and Dubos, 2014).

To derive the momentum equations we follow Holm et al. (1998) variational derivation and adapt it to the case of non-orthogonal curvilinear coordinates. We start from the Lagrangian

$$
\left.\mathcal{L}\left(U_{i}, \rho, b, p\right)=\iiint\left[\rho\left(\frac{1}{2} u_{i} U_{i}+b z\right)\right)-p\left(\frac{\rho}{\rho_{0}}-1\right)\right] \mathcal{V} d \xi d \eta d \sigma,
$$

where the index notation $i=1,2,3$,stands for $(\xi, \eta, \sigma), U_{i}$ 's are the components of the flux $\mathbf{U}, u_{i}$ 's are the component of the momentum $\mathbf{u}$ and $b$ is the buoyancy relative to the Boussinesq reference density $\rho_{0}$. The variations of the Lagrangian are

$$
\begin{aligned}
\frac{1}{\rho} \frac{\delta \mathcal{L}}{\delta U_{i}} & =u_{i} \\
\frac{1}{\rho} \frac{\delta \mathcal{L}}{\delta b} & =z \\
\frac{\delta \mathcal{L}}{\delta \rho} & =\frac{1}{2} u_{i} U_{i}+b z-\frac{p}{\rho_{0}} \\
\frac{\delta \mathcal{L}}{\delta p} & =\frac{\rho}{\rho_{0}}-1 .
\end{aligned}
$$

600 The total pressure $p=p^{h}+q$ arises as a Lagrange multiplier enforcing 601 the incompressibility constraint $\rho=\rho_{0}$. The Euler Poincaré equation, or 
602

momentum equation, reads (Holm et al., 1998)

$$
\partial_{t} \mathbf{u}+\operatorname{curl} \mathbf{u} \times \mathbf{U}+\boldsymbol{\nabla}(\mathbf{u} \cdot \mathbf{U})-\nabla \frac{\delta \mathcal{L}}{\delta \rho}+\frac{1}{\rho} \frac{\delta \mathcal{L}}{\delta b} \nabla b=0
$$

603

$$
\partial_{t} \mathbf{u}+\operatorname{curl} \mathbf{u} \times \mathbf{U}=-\boldsymbol{\nabla}\left(\frac{1}{2} \mathbf{U} \cdot \mathbf{u}+\frac{p}{\rho_{0}}\right)+b \boldsymbol{\nabla} z .
$$

${ }_{604}$ (C.4) is the momentum equation in vector-invariant form. It is identical to 605 Tort and Dubos (2014). The formalism comes with the tracer conservation equation $\partial_{t} \phi+\operatorname{div}(\mathbf{U} \phi)=0$, that holds for any tracer. In particular it translates into the continuity equation (17) for $\phi=\rho$. We now show how (C.4) can be transformed into the flux form (21) plus a pseudo force $\tilde{\mathbf{G}}$. The idea is to prove that

$$
\left[\operatorname{curl} \mathbf{u} \times \mathbf{U}+\nabla\left(\frac{1}{2} \mathbf{U} \cdot \mathbf{u}\right)\right] \mathcal{V}=\partial_{j}\left(\mathcal{A}_{j} U_{j} \mathbf{u}\right)-\tilde{\mathbf{G}} \mathcal{V}
$$

610 In components the curl term reads

$$
\begin{aligned}
\left.\mathcal{V}(\operatorname{curl} \mathbf{u} \times \mathbf{U})\right|_{i} & =\epsilon_{i j k} \epsilon_{j l m} \partial_{\nu}\left(u_{m} h_{m}\right) U_{k} \frac{\mathcal{A}_{k}}{h_{i}} \\
& =\left(\delta_{k l} \delta_{i m}-\delta_{k m} \delta_{i l}\right) \partial_{l}\left(u_{m} h_{m}\right) U_{k} \frac{\mathcal{A}_{k}}{h_{i}} \\
& =\partial_{k}\left(U_{k} \mathcal{A}_{k} u_{i}\right)-U_{k} \mathcal{A}_{i} \partial_{i} u_{k}+U_{k} \frac{\mathcal{A}_{k}}{h_{i}}\left(u_{i} \partial_{k} h_{i}-u_{k} \partial_{i} h_{k}\right),
\end{aligned}
$$

611 where we have used $\partial_{k}\left(U_{k} \mathcal{A}_{k}\right)=0$. The next step is to rearrange

$$
\begin{aligned}
\left.\mathcal{V} \boldsymbol{\nabla}\left(\frac{1}{2} \mathbf{U} \cdot \mathbf{u}\right)\right|_{i} & =\frac{1}{2} \mathcal{A}_{i} \partial_{i}\left(U_{k} u_{k}\right) \\
& =\mathcal{A}_{i} U_{k} \partial_{i} u_{k}+\frac{1}{2} \mathcal{A}_{i}\left(u_{k} \partial_{i} U_{k}-U_{k} \partial_{i} u_{k}\right) \\
& =\mathcal{A}_{i} U_{k} \partial_{i} u_{k}+\frac{1}{2} \mathcal{A}_{i} u_{k} u_{j} \partial_{i} T_{j k}
\end{aligned}
$$


612

where we have used the property

$$
\frac{1}{h_{\xi}} \partial_{\xi} s_{\eta}-\frac{1}{h_{\eta}} \partial_{\eta} s_{\xi}=\frac{1}{h_{\xi} h_{\eta}}\left(s_{\xi} \partial_{\eta} h_{\xi}-s_{\eta} \partial_{\xi} h_{\eta}\right) .
$$

Summing (C.6) and (C.7) gives (C.5) with the general expression for the

$$
\tilde{\mathbf{G}}_{i}=\frac{U_{k}}{h_{i} h_{k}}\left(u_{k} \partial_{i} h_{k}-u_{i} \partial_{k} h_{i}\right)-\frac{1}{2 h_{i}} u_{k} u_{j} \partial_{i} T_{j k} .
$$

Using the expression for $T_{i j}$ allows to explicit each component

$$
\begin{aligned}
& \tilde{G}_{u}=+\left(v \partial_{\xi} h_{\eta}-u \partial_{\eta} h_{\xi}\right) \frac{V}{h_{\xi} h_{\eta}}+\frac{w W}{h_{\xi} h_{\sigma}} \partial_{\xi} h_{\sigma}+\frac{w U}{h_{\xi}} \partial_{\xi} s_{\xi}+\frac{w V}{h_{\xi}} \partial_{\xi} s_{\eta} \\
& \tilde{G}_{v}=-\left(v \partial_{\xi} h_{\eta}-u \partial_{\eta} h_{\xi}\right) \frac{U}{h_{\xi} h_{\eta}}+\frac{w W}{h_{\eta} h_{\sigma}} \partial_{\eta} h_{\sigma}+\frac{w U}{h_{\eta}} \partial_{\eta} s_{\xi}+\frac{w V}{h_{\eta}} \partial_{\eta} s_{\eta} \\
& \tilde{G}_{w}=0
\end{aligned}
$$

with

$$
\begin{aligned}
& G_{u}=+\left(V \partial_{\xi} h_{\eta}-U \partial_{\eta} h_{\xi}\right) \frac{V}{h_{\xi} h_{\eta}} \\
& G_{v}=-\left(V \partial_{\xi} h_{\eta}-U \partial_{\eta} h_{\xi}\right) \frac{U}{h_{\xi} h_{\eta}},
\end{aligned}
$$

\footnotetext{
The pseudo-force differs from the hydrostatic case by the presence of terms ${ }_{621}$ proportional to $w$, the vertical momentum. They vanish in the hydrostatic 622 case because the vertical momentum is identically zero.

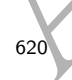


623 642 643

Acknowledgments. We thank Labex Mer for supporting this study and the two reviewers, Francis Auclair and an anonymous one, for helping improving the clarity of the paper.

\section{References}

M. Nikurashin, G. K. Vallis, A. Adcroft, Routes to energy dissipation for geostrophic flows in the Southern Ocean, Nature Geoscience 6 (1) (2013) $48-51$.

M. J. Molemaker, J. C. McWilliams, W. K. Dewar, Submesoscale instability and generation of mesoscale anticyclones near a separation of the California Undercurrent, Journal of Physical Oceanography 45 (3) (2015) 613-629.

J. Callies, R. Ferrari, J. M. Klymak, J. Gula, Seasonality in submesoscale turbulence, Nature communications 6 .

J. Gula, M. J. Molemaker, J. C. McWilliams, Gulf Stream dynamics along the southeastern US seaboard, Journal of Physical Oceanography 45 (3) (2015) 690-715.

P. P. Sullivan, J. C. McWilliams, C.-H. Moeng, A subgrid-scale model for large-eddy simulation of planetary boundary-layer flows, Boundary-Layer Meteorology 71 (3) (1994) 247-276.

J. Marshall, C. Hill, L. Perelman, A. Adcroft, Hydrostatic, quasi-hydrostatic, and nonhydrostatic ocean modeling, J. Geophys. Res. 102 (1997) 57335752. 
Y. Kanarska, V. Maderich, A non-hydrostatic numerical model for calculating free-surface stratified flows, Ocean Dynamics 53 (3) (2003) 176-185.

Y. Kanarska, A. Shchepetkin, J. McWilliams, Algorithm for non-hydrostatic dynamics in the regional oceanic modeling system, Ocean Modelling 18 (3) (2007) 143-174.

F. Auclair, C. Estournel, J. W. Floor, M. Herrmann, C. Nguyen, P. Marsaleix, A non-hydrostatic algorithm for free-surface ocean modelling, Ocean modelling 36 (1) (2011) 49-70.

K. Klingbeil, H. Burchard, Implementation of a direct nonhydrostatic pressure gradient discretisation into a layered ocean model, Ocean Modelling 65 (2013) 64-77.

W. C. Skamarock, J. B. Klemp, A time-split nonhydrostatic atmospheric model for weather research and forecasting applications, Journal of Computational Physics 227 (7) (2008) 3465-3485.

E. H. Müller, R. Scheichl, Massively parallel solvers for elliptic partial differential equations in numerical weather and climate prediction, Quarterly Journal of the Royal Meteorological Society 140 (685) (2014) 2608-2624.

S. Williams, A. Waterman, D. Patterson, Roofline: an insightful visual performance model for multicore architectures, Communications of the ACM 52 (4) (2009) 65-76.

A. F. Shchepetkin, J. C. McWilliams, A method for computing horizontal pressure-gradient force in an oceanic model with a nonaligned vertical coordinate, Journal of Geophysical Research: Oceans 108 (C3). 
N 687

M. A. Taylor, A. Fournier, A compatible and conservative spectral element method on unstructured grids, Journal of Computational Physics 229 (17) (2010) 5879-5895.

M. Desbrun, E. Kanso, Y. Tong, Discrete differential forms for computational modeling, in: Discrete differential geometry, Springer, 287-324, 2008.

J. Thuburn, C. J. Cotter, A framework for mimetic discretization of the rotating shallow-water equations on arbitrary polygonal grids, SIAM Journal on Scientific Computing 34 (3) (2012) B203-B225

M. J. Molemaker, J. C. McWilliams, I. Yavneh, Baroclinic instability and loss of balance, Journal of Physical Oceanography 35 (9) (2005) 1505-1517.

T. Dubos, S. Dubey, M. Tort, R. Mittal, Y. Meurdesoif, F. Hourdin, DYNAMICO, an icosahedral hydrostatic dynamical core designed for consistency and versatility, Geoscientific Model Development Discussions 8 (2) (2015) 1749-1800.

A. J. Chorin, Numerical solution of the Navier-Stokes equations, Mathematics of computation 22 (104) (1968) 745-762.

J. B. Bell, P. Colella, H. M. Glaz, A second-order projection method for the incompressible Navier-Stokes equations, Journal of Computational Physics 85 (2) (1989) 257-283.

L. J. Grady, J. Polimeni, Discrete calculus: Applied analysis on graphs for computational science, Springer Science \& Business Media, 2010. 
${ }_{688}$ M. Tort, T. Dubos, Dynamically consistent shallow-atmosphere equations ${ }_{689}$ with a complete Coriolis force, Quarterly Journal of the Royal Meteoro$690 \quad$ logical Society 140 (684) (2014) 2388-2392.

D. D. Holm, J. E. Marsden, T. S. Ratiu, The Euler-Poincaré equations ${ }_{692}$ and semidirect products with applications to continuum theories, Adv. 693 in Math. 137 (1998) 1-81. 